\title{
Comprehensive comparison of two tracking codes for single-particle dynamics evaluation of a next-generation storage ring light source
}

\author{
Michael Borland $\odot,{ }^{1, *}$ Yipeng Sun, ${ }^{1}$ and Xiaobiao Huang ${ }^{2, \dagger}$ \\ ${ }^{1}$ Argonne National Laboratory, 9700 Cass Avenue, Lemont, Illinois 60439, USA \\ ${ }^{2}$ SLAC National Accelerator Laboratory, 2575 Sand Hill Road, Menlo Park, California 94025, USA
}

(Received 10 April 2019; published 7 November 2019)

\begin{abstract}
Next-generation light sources based on the multibend achromat concept place great reliance on the accuracy of beam dynamics modeling. To ensure the success of such light sources, confirmation of design performance with at least two independent codes is prudent. The APS Upgrade (APS-U) lattice has been designed using the code ELEGANT, which has existing and new features that are needed by the design. Corresponding, independent improvements have recently been made to the accelerator toolbox (AT), in order to permit an independent check of nonlinear dynamics predictions. The new developments include the modeling of combined-function bending magnets with straight geometry and improved fringe field effects of quadrupole magnets. Calculations of linear and nonlinear lattice parameters and particle tracking results were compared, showing remarkable agreement was found between the two independently developed codes. This provides significant confirmation of the feasibility of the APS-U design.
\end{abstract}

DOI: $10.1103 /$ PhysRevAccelBeams.22.114601

\section{INTRODUCTION}

Accurate lattice modeling is of crucial importance in storage ring lattice designs. The successful operation of a storage ring requires sufficient dynamic acceptance (DA) and local momentum acceptance (LMA) throughout the ring. During the design phase, these nonlinear beam dynamics measures are obtained through particle tracking with a lattice model. Presently a part of the standard design practice is to optimize the tracked DA and LMA with multiobjective optimizers using linear lattice parameters and nonlinear magnets as optimization decision variables [1-3]. Because the storage ring nonlinear beam dynamics depends on the linear and nonlinear lattice parameters in complex, subtle ways, small errors in the lattice model in either linear or nonlinear optics could potentially cause large errors in the DA and LMA predictions. Therefore, the validity of the multiobjective optimization results may strongly depend on the accuracy of the lattice model. Recent work of experimental optimization of storage ring nonlinear dynamics has led to substantial improvement in the dynamic aperture or momentum apertures [4-7], which may serve as an indication that model-based machine settings need improvement. This is particularly important

\footnotetext{
*borland@anl.gov

xiahuang@slac.stanford.edu
}

Published by the American Physical Society under the terms of the Creative Commons Attribution 4.0 International license. Further distribution of this work must maintain attribution to the author(s) and the published article's title, journal citation, and DOI. when fast commissioning is required, as is the case for the APS Upgrade (APS-U) [8].

The requirement for high accuracy lattice modeling has increased substantially in recent years as many labs began pushing for the next generation, multibend-achromat (MBA) based storage ring light sources [8-10]. These new rings utilize much stronger quadrupole magnets to pack many more lattice cells over the circumference as compared to a traditional third generation storage ring. Chromaticity correction of such rings requires much stronger sextupoles, despite the adoption of the hybrid-MBA cell, a creative lattice configuration that reduces the sextupole strengths significantly [9]. The increase of the numbers and strengths of magnets makes it more important to model the individual magnets accurately since the combined errors from all magnets could add up to large errors in the nonlinear beam dynamics performance prediction.

The new lattice features of the next generation storage ring light sources, such as longitudinal gradient dipoles [11-15], combined-function bending magnets with strong quadrupole components, and negative bending [16,17], call for new developments in the lattice codes. Combined-function bending magnets with straight geometry have been used in many third generation light sources. There have been a few studies on the lattice effects of such magnets [18-20]. But these studies have not yielded symplectic models that are usable in tracking codes. Fringe field effects are a major source of discrepancy between the lattice model and a real machine. Traditionally quadrupole magnets are often modeled as hard edge elements in lattice codes, although the impact of fringe fields to linear and nonlinear optics has been previously studied theoretically [21-24]. 
Because the success of next-generation storage ring light source projects, such as the APS-U, relies heavily on lattice modeling, it is important to ensure the lattice modeling codes are implemented correctly. One way to check the validity of the codes is to benchmark against each other with lattice models that include all of the critical features. About 9 years ago, the storage ring light source community conducted an exercise to benchmark the codes that are commonly used for storage ring modeling [25]. A wide spread of results in both linear and nonlinear lattice parameters was found among the codes, which suggested that placing too much confidence in any one code was inappropriate.

Recently we have implemented new features for ELEGANT [26] and the accelerator toolbox (AT) [27] in separate, independent efforts. These features address several of the issues noted above that are particular to nextgeneration storage rings. Afterwards we benchmarked the two codes using the APS-U lattice model and found very good agreement. In this paper we describe the new developments in both codes and the benchmarking results. We hope our effort will help the community to converge to a common lattice modeling practice. This paper is organized as follows. In Sec. II, the new features of both codes are described. Section III presents the various benchmarking results. The conclusion is given in Sec. IV.

\section{NEW SINGLE PARTICLE DYNAMICS FEATURES}

To meet the lattice modeling needs for the APS-U project, recently several new features were added to the ELEGANT code. The single particle dynamics features to be discussed here include the exact drift space, the quadrupole fringe field, and the combined-function dipole magnets with straight geometry. In order to provide an independent benchmark and provide further confidence in modeling of APS-U, the AT code has been recently updated to add some of these features. The new single particle dynamics features in ELEGANT and AT are summarized in Table I.

\section{A. Exact drift space}

The Hamiltonian of ultra-relativistic particle motion in a drift space in canonical coordinates $\left(x, p_{x}, y, p_{y}, z, \delta\right)$ is given by

$$
H=-\sqrt{(1+\delta)^{2}-p_{x}^{2}-p_{y}^{2}}+(1+\delta) .
$$

Some tracking codes implement a simplified model for the drift space, using the Hamiltonian

$$
H=\frac{p_{x}^{2}+p_{y}^{2}}{2(1+\delta)},
$$

which is obtained from Eq. (1) by expanding the terms under the square root. The simplified model does not need to compute the square roots in the map, but it lacks the higher order effects.

ELEGANT provides the exact drift space model described by Eq. (1) through the element type EDRIFT. Elements that use drift-kick-drift type symplectic integration in ELEGANT, such as KQUAD and KSEXT, also implement the exact drift space. AT has implemented the exact drift space with the pass method LaDrift. Its new fourth order symplectic integrator, StrMPoleSymplectic4NPass, uses the exact drift space, along with other features to be described below.

It turns out the conversion to exact drift space does not make a significant impact to the nonlinear beam dynamics behavior in the case of the APS-U lattice. For example, comparisons were performed using ELEGANT of DA and LMA using exact and approximate drift-space implementations for the field-free regions (i.e., regions external to dipoles, quadrupoles, and sextupoles). No significant differences were seen.

\section{B. Combined-function bending magnets with straight geometry}

Combined-function dipole-quadrupole magnets are commonly used in storage ring light sources to save space and to modify the horizontal damping partition. If the magnet is built on a curved geometry that follows the reference trajectory of the ideal particle, it can be modeled as a sector dipole, with the fields expanded in curvilinear coordinates [28]. The linear motion can be described with a transfer matrix for which analytical expressions exist [29]. If higher-order multipole components are involved, symplectic integration in the curved reference system can be performed. For example, in ELEGANT this is performed using the CSBEND element, which uses the exact Hamiltonian and a fourth-order symplectic integrator.

However, some combined dipole-quadrupole magnets are built on a straight geometry, for reasons of mechanical

TABLE I. New single particle dynamics features in ELEGANT and AT. (Note that the KQUAD and KSEXT elements have been standard in elegant for many years.)

\begin{tabular}{lccl}
\hline \hline Type & ELEGANT & AT & \multicolumn{1}{c}{ Comments } \\
\hline Drift & EDRIFT & LaDrift & Exact drift space \\
Dipole & CCBEND & BndStrMPoleSymplectic4Pass & Straight dipole \\
Quadrupole & KQUAD & StrMPoleSymplectic4NPass & w/quadrupole fringe field \\
Sextupole & KSEXT & StrMPoleSymplectic4NPass & Using exact drift \\
\hline \hline
\end{tabular}




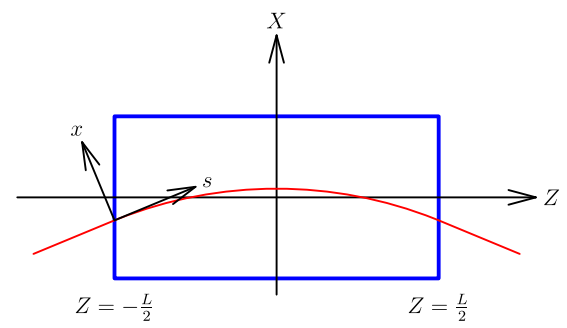

FIG. 1. Coordinates in a straight geometry combined-function dipole magnet.

simplicity and cost. In APS-U, two families of combinedfunction reverse bending magnets (Q4 and Q5) will have straight geometry. In this case, the field expansion used for a curved-pole magnet is incorrect. However, the magnetic field in the magnets can be given in very simple form using the Cartesian coordinates $(X, Y, Z)$ by (see Fig. 1)

$$
B_{Y}=B_{0}+B_{1} X, \quad B_{X}=B_{1} Y .
$$

In such a magnet, the reference trajectory is not an arc of a circle, since the bending field varies along the trajectory. The focusing gradient also varies with the beam trajectory as the component of the focusing gradient on the transverse plane will change with the $s$-coordinate. Therefore, this type of magnet cannot be described by the sector dipole model.

Modeling of a straight dipole magnet can be broken into three parts. First, at the entrance of the magnet, a coordinate transformation is performed to the Cartesian coordinates with one axis parallel to the axis of the magnet. Second, we must model the body of the magnet, for which,because of the potentially large values of the Cartesian coordinates, the exact Hamiltonian must be used. This is similar in principle to modeling of straight elements like quadrupoles and sextupoles using the exact Hamiltonian, something that has been standard in ELEGANT for many years. Third, at the exit face an inverse transformation is performed to go back to the usual coordinates. This is similar to an approach proposed in Ref. [30] and was recently implemented in ELEGANT [31] as the CCBEND element, then in AT.

While the implementations in ELEGANT and AT are based on the same concepts, they were performed independently. For simplicity, we'll describe the AT implementation. We solve the beam motion through symplectic integration in the Cartesian coordinate system, using coordinates $\left(X, X^{\prime}=\frac{d X}{d Z}, Y, Y^{\prime}=\frac{d Y}{d Z}, z, \delta\right)$, where $z$ is path length difference with the reference particle. (ELEGANT, by contrast, works with canonical coordinates, as detailed in [31].) At the entrance face, the coordinate transformation consists of three steps. First, the angle coordinates are found with

$$
x^{\prime}=\frac{d x}{d s}=\frac{p_{x}}{\sqrt{(1+\delta)^{2}-p_{x}^{2}-p_{y}^{2}}},
$$

$$
y^{\prime}=\frac{d y}{d s}=\frac{p_{y}}{\sqrt{(1+\delta)^{2}-p_{x}^{2}-p_{y}^{2}}} .
$$

The particles are then propagated from the $x y$ plane to the $X Y$ plane at the entrance point, which is followed by a rotation transformation to the Cartesian coordinates. The last two steps combined can be expressed as

$$
\begin{gathered}
X=\frac{x \cos \psi}{\cos \left(\frac{\theta}{2}+\psi\right)}+X_{0}, \\
X^{\prime}=\tan \left(\frac{\theta}{2}+\psi\right), \\
Y^{\prime}=\frac{y^{\prime}}{\cos \frac{\theta}{2}-x^{\prime} \sin \frac{\theta}{2}}, \\
Y=y+x Y^{\prime} \sin \frac{\theta}{2}, \\
\Delta s=\frac{x \tan \frac{\theta}{2} \sqrt{1+x^{\prime 2}+y^{\prime 2}}}{1-x^{\prime} \tan \frac{\theta}{2}},
\end{gathered}
$$

where $\theta$ is the bending angle of the magnet, $\frac{\theta}{2}$ is assumed to be the entrance angle, $\psi=\tan ^{-1} x^{\prime}$, and $X_{0}$ is the $X$-coordinate for the reference orbit at the entrance point (where $Z=-\frac{L}{2}, L$ is the straight length of the magnet). At the exit face, a similar transformation is performed. These transformations were previously used in AT for the pass method for direct integration of the equation of motion through arbitrary magnetic fields [19].

In both codes, the integration through the body of the rectangular magnet is done with the fourth order symplectic integrator, using drift and kick maps. The exact map for drift spaces has to be used in this case, given the large angle coordinates in the Cartesian coordinate system.

Unlike the case of a curvilinear dipole, the reference trajectory through a straight dipole must be determined numerically. Because the bending field in the magnet varies with the $X$-coordinate, the bending angle of a particle depends on the entrance point. The entrance point for the reference orbit, $X_{0}$, needs to be found numerically using the condition that the reference orbit is symmetric about the middle of the magnet, with a total bending angle of $\theta$. However, this might result in a trajectory that is not centered in the magnet and so does not use the good field region. If in addition the magnetic field strength is varied (i.e., scaling $B_{0}$ and $B_{1}$ proportionally), the entrance point for the reference orbit will change. This gives us the freedom to set the reference orbit through the center of the good field region. This tuning procedure is implemented automatically in ELEGANT and AT. When the magnetic field profile is available, from simulation or measurements, the current set point of the magnet should be determined according to the alignment requirement using a similar numeric procedure [18]. 


\section{Dipole fringe field}

Dipole fringe fields are a complex and potentially difficult subject for storage ring modeling. We've explored these effects for the APS-U lattice (described in more detail in Sec. III) using ELEGANT, which incorporates a number of fringe field options into the CSBEND element. The CCBEND element, in contrast, has less sophisticated fringe field modeling; however, the bending radii for the Q4 and Q5 reverse-bend magnets are $90 \mathrm{~m}$ and $124 \mathrm{~m}$, respectively, which makes this less of a concern. In contrast, some of the segments of the longitudinal gradient dipoles have bending radii as short as $31 \mathrm{~m}$, so it is prudent to assess the possible effects.

As indicated above, most of the transverse-gradient dipoles (excepting Q4 and Q5) in the APS-U lattice are in fact curved and are correctly modeled with the CSBEND element in ELEGANT (and the equivalent in AT). This is particularly important for the dipoles with strong transverse gradients. The five-segment longitudinal gradient dipoles are straight-pole magnets, but are modeled at present as a series of CSBEND elements with edge angles that reflect the actual path of the beam across the segment boundaries. This is a reasonable approach because there are ideally no transverse gradients, though we will return to this point below.

The fringe field options for CSBEND include both nonsymplectic and symplectic models, of which only the latter are of interest here. Among these is a symplectic variation of [29], in which we apply momentum kicks according to

$$
\begin{gathered}
\Delta p_{x}=\frac{x \tan \alpha}{\rho_{0}} \\
\Delta p_{y}=-\frac{y \tan \left(\alpha-\frac{\psi}{1+\delta}\right)}{\rho_{0}}
\end{gathered}
$$

where $\alpha$ is the edge angle, $\rho_{0}$ is the nominal bending radius, and $\psi$ is a soft-fringe parameter defined in [29]. We will refer to this as the KBS1 model. A variant of this model (KBS0), uses approximate forms of Eqs. (4), e.g., $x^{\prime}=$ $p_{x} /(1+\delta)$, corresponding largely to the linear matrix method described in [29].

A second, more recent approach is also available [32], in two variants. In the first variant (KH1), only linear terms are included in the transverse coordinates, while the momentum offset is included to all orders. In the second variant (KH2), all terms described in [32] are included; this results in orbit distortion due to the extension of the fringe fields outside the body of the magnet, which can be suppressed in ELEGANT (and in reality) by adjusting the overall strength of the magnet.

To understand the extent to which these models matter, we compared the tunes and chromaticities for the four models. In the absence of soft-fringe effects, the models
TABLE II. Illustration of the potential effect of the fringe field model for CSBEND elements on the tunes and chromaticities.

\begin{tabular}{lccrc}
\hline \hline Model & $\nu_{x}$ & $\nu_{y}$ & $\xi_{x}$ & $\xi_{y}$ \\
\hline KBS0 & 95.0999 & 36.0931 & 8.118 & 4.706 \\
KBS1 & 95.0993 & 36.0925 & 8.114 & 4.708 \\
KH1 & 95.2493 & 36.2192 & 10.510 & 6.088 \\
KH2 & 95.2990 & 35.7889 & 9.166 & 6.684 \\
\hline \hline
\end{tabular}

give nearly identical results for the momentum-dependent tunes. To understand the extent to which soft-fringe effects matter, we assumed that the half-gap of all the dipole magnets was $13 \mathrm{~mm}$ and that the fringe integral $K$ defined in [29] was 0.5. As Table II shows, both the tunes and the chromaticities for the KH1 and KH2 models differ considerably from the KBS0 and KBS1 models and each other. The magnitude of the differences are perhaps surprising, but we of course the assumed soft-fringe parameters are somewhat arbitrary. We nevertheless compared the momentum-dependent tune footprint for the four models after correction of the tunes (using the Q1 and Q2 families) and chromaticities (using two families composed of $\mathrm{S} 1 / \mathrm{S} 3$ and $\mathrm{S} 2$ magnets). The results, shown in Fig. 2, suggest that softfringe effects are readily compensated.

Since the momentum dependence of the tunes is not identical in the four cases, refining these models for the APS-U design will be worthwhile beyond allowing the tunes and chromaticities to be more accurately predetermined. To begin, we require a more sophisticated approach to determining the fringe parameters than we have used for this illustration. This starts with computation of the actual fringe parameters from computed field maps, followed by fine-tuning through comparison with the results of tracking through the field maps. The longitudinal gradient dipoles

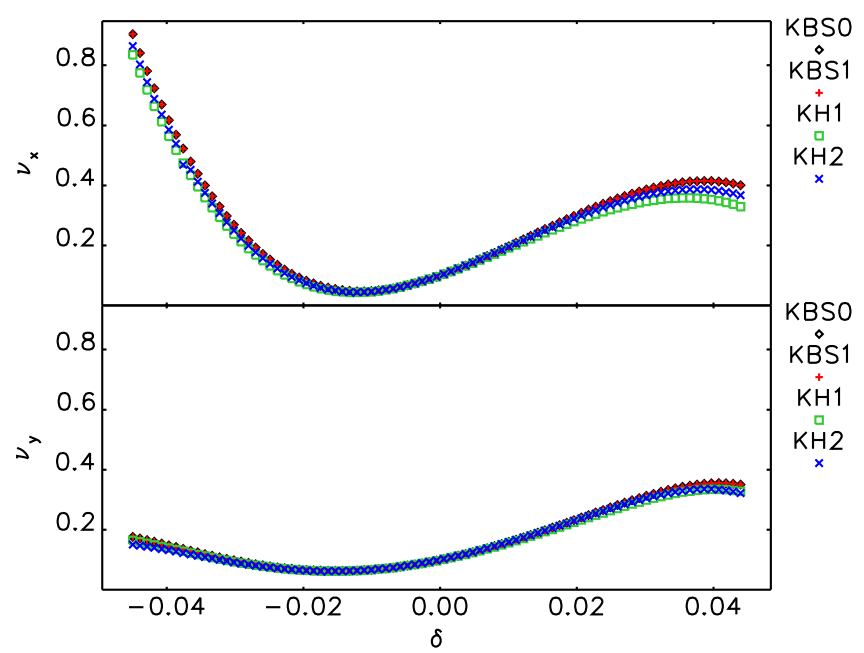

FIG. 2. Momentum-dependence of tunes from tracking with various bending magnet fringe models, after correction of the tunes and linear chromaticities. 
are particularly challenging, in part because the field between the segments does not drop to zero, contrary to what is assumed here. In addition, because these are straight magnets with finite pole widths, there are sextupole terms in the body of the magnets and sextupolelike terms in the exterior and interior fringes. Owing to the curved trajectory of the beam through the straight magnet, these terms result in focusing effects that are not in the present model. Inclusion of these effects is the subject of ongoing work based on computed 3D field maps. The planned approach will use CCBEND elements for each segment, with body and edge sextupole terms included and with transverse offsets that reflect the trajectory of the beam through the straight magnet.

Pending completion of this analysis, we have assumed in the remainder of this paper that soft-edge effects on dipoles can be ignored, and have also assumed that the longitudinal gradient dipoles can be modeled as a series of flat-field elements with the CSBEND approach. The default KBS0 model is also used for the remainder of this paper; it gives nearly identical results to the other models when soft-fringe effects are not included.

\section{Quadrupole fringe field}

The magnetic field of a realistic quadrupole magnet extends beyond the iron core through a smooth transition curve at both edges, while in lattice models the magnet is typically described with a hard-edge field model in which the magnetic field drops to zero abruptly. The hard-edge model is not physically self-consistent, yet it has been remarkably successful in the past. For the new type of rings with more and stronger quadrupoles, the errors from the hard-edge model need to be properly accounted for. The linear optics error comes from the difference in the distribution of the focusing gradient between the real and the hard-edge profiles. This "soft fringe field" effect has been studied in Ref. [22] and later in more detail in Ref. [23]. There are also nonlinear effects from the quadrupole fringe field. [22,24].

In AT the fringe field model in Ref. [22] was previously implemented for the transfer-matrix-based quadrupole pass method. Recently the more accurate model of Ref. [23] that was implemented in ELEGANT was also implemented in AT, both for the transfer matrix based pass method and the straight element symplectic integrator. Suppose the difference of the focusing gradient profiles between the real and the hard-edge models is given by a function

$$
\Delta K(s)=K(s)-K_{h}(s),
$$

where $K(s)$ is the actual focusing gradient and $K_{h}(s)$ is the focusing gradient for the corresponding hard-edge model. The function $K_{h}(s)$ is zero for $s<-s_{0}$ or $s>s_{0}$ and is equal to $K_{0}$ for $-s_{0} \leq s \leq s_{0}$, where $K_{0}$ is the normalized gradient for the quadrupole, $s_{0}=\frac{L}{2}$, and $L$ is the effective

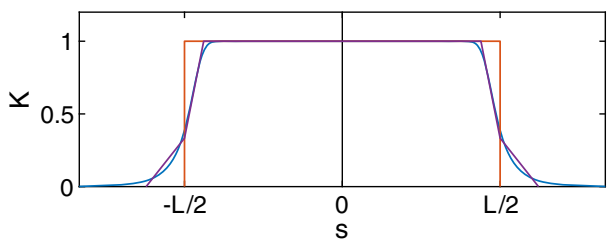

FIG. 3. Focusing gradient profile $K(s)$ for a realistic quadrupole magnet (blue) and the hard-edge model (red). The purple profile is the bilinear profile for testing the fringe field modeling approach.

length of the magnet. The fringe field model in Ref. [23] characterize the function $\Delta K(s)$ with a series of integrals for both the entrance and exit edges.

Figure 3 shows an example of the focusing gradient profile of a real quadrupole magnet and its hard-edge model. The gradient of the hard-edge model is equal to the average gradient around the center of the magnet and the effective length is chosen to make the integrated gradient of the hard edge model equal to that of the real magnet. In both the ELEGANT and AT implementations, the actual fringe field integrals as defined in Ref. [23] are supplied as parameters to the quadrupole element. At the entrance and exit edges, these integrals are used to modify the canonical coordinates according to the corresponding Hamiltonian terms.

The quadrupole fringe field modeling is checked with a bilinear test fringe field profile. In the test profile the gradient decreases to zero in two linear slopes, with a change of slope at the hard edge boundary, as illustrated in Fig. 3 (purple curve). Defining parameter $\Delta$ as the distance between the hard edge boundary to the starting point of the slope in the magnet, the fringe integrals for the bilinear profile are calculated to be

$$
\begin{gathered}
I_{0 p}=-I_{0 m}=\frac{1}{3} K_{0} \Delta, \\
I_{1 p}=2 I_{1 m}=\frac{2}{9} K_{0} \Delta^{2}, \\
I_{2 p}=-4 I_{1 m}=\frac{2}{9} K_{0} \Delta^{3}, \\
I_{2 p}=8 I_{1 m}=\frac{4}{15} K_{0} \Delta^{4}, \\
\Lambda_{2}^{+}=2 \Lambda_{2}^{-}=\frac{4}{135} K_{0}^{2} \Delta^{3} .
\end{gathered}
$$

The transfer matrix for the quadrupole magnet obtained with the quadrupole fringe field model can be compared to the one obtained by slicing the gradient profile into many pieces and concatenating the hard-edge transfer matrices of all pieces. In a numerical test, where $L=1.0 \mathrm{~m}$, $\Delta=0.1 \mathrm{~m}$, and $K_{0}=1.5 \mathrm{~m}^{-2}$, the differences between the transfer matrices obtained with the two methods in the 
above are

$$
\begin{aligned}
& \Delta R_{x}=\left(\begin{array}{cc}
1.4 & -5.8 \\
-7.2 & 1.4
\end{array}\right) \times 10^{-6}, \\
& \Delta R_{y}=\left(\begin{array}{ll}
-2.3 & -8.3 \\
-5.9 & -2.3
\end{array}\right) \times 10^{-6},
\end{aligned}
$$

while if using the hard-edge model without the quadrupole fringe field effect, the differences are

$$
\begin{aligned}
& \Delta R_{x}=\left(\begin{array}{cc}
-0.0005 & 0.0079 \\
0.0113 & -0.0005
\end{array}\right), \\
& \Delta R_{y}=\left(\begin{array}{cc}
-0.0008 & -0.0136 \\
0.0181 & -0.0008
\end{array}\right),
\end{aligned}
$$

where $\Delta R_{x, y}$ are differences for the horizontal and vertical transfer matrices, respectively.

Additional numerical tests were performed with realistic fringe profiles and similar agreement between the fringe field model and the slicing approach was observed. In one test performed with ELEGANT for one of the doublet magnets in the APS-U lattice, comparison was made to symplectic tracking through quadrupole fields defined by a generalized gradient expansion [33] computed from OPERA-generated field data. Such tracking is available using the BGGEXP element. Agreement in the linear matrix elements was within $2.5 \times 10^{-7}$, while agreement with the second-order matrix elements was within $5.0 \times 10^{-4}$. In performing this comparison, it was found to be essential to properly define $K(s)$ using the first term from the generalized gradient expansion, $2 C_{2, s}^{(0)}(z)$. Analysis based on zdependent harmonic analysis of the OPERA data showed much poorer agreement.

The leading terms in the nonlinear quadrupole fringe field effects for quadrupoles is the hard-edge map studied in Ref. [24]. The map for a normal quadrupole is not easy to implement in a tracking code since it is not a kick map. However, as pointed out in Ref. [24], the hard-edge nonlinear map of a skew quadrupole, whose generating function is given by

$$
f=\frac{a_{1}}{6}\left(x^{3} p_{y}+y^{3} p_{x}\right), \quad a_{1}=\frac{1}{B \rho} \frac{\partial B_{x}}{\partial x},
$$

is composed of two kick maps. Therefore, the map for a normal quadrupole can be modeled by first rotating the transverse coordinates of the particles by $45^{\circ}$, applying the two kicks, and then rotating backward by $45^{\circ}$. In AT, this is implemented at both edges of the element for the updated fourth order symplectic integrator pass method.

For the nonlinear effects, ELEGANT uses the formulation of Lee-Whiting [34], which involves changes to position and momentum coordinates at the entrance and exit of the quadrupole. Higher order terms are also included [35], but these have a negligible effect on beam dynamics. As argued by Forest [30], the momentum kicks and position "jumps" are of comparable magnitude and should both be included, or else the map is "grossly nonsymplectic". This implementation and the importance of including both kicks and jumps was validated using comparison with the BGGEXP element to determine transport matrices up to third order by performing fits to tracking data [36]. We found that discrepancies in the third-order tracking-derived matrix were reduced by three orders of magnitude by inclusion of these terms. Removal of the position jumps significantly worsened the agreement.

\section{COMPARISON BETWEEN THE TWO CODES WITH APS-U LATTICE}

Comparison of the two codes, ELEGANT and AT, was conducted as a way to validate the implementation of the modeling methods, including those described above as well as others needed for modeling of storage rings. We used the APS-U storage ring lattice as the test model. The APS-U lattice consists of 40 hybrid MBA cells over a circumference of $1103.6 \mathrm{~m}$. The lattice cell contains five families of pure quadrupoles (Q1, Q2, Q3, Q6, and Q7), three families of combined-function sector dipoles (M3, M4 and Q8), two families of longitudinal gradient dipoles (M1 and M2), and two families of straight geometry combinedfunction dipoles (Q4 and Q5). Each family of magnets in the above has two magnets in one cell, which are symmetrically placed about the cell center, except for sector dipole M4 as it is located right at the center. There are six sextupoles in each cell, placed in reflection-symmetric locations of each cell in the high-dispersion regions. There are no harmonic sextpoles or powered octupoles, although provision for octupoles in dispersive and nondispersive locations has been made. Instead, multiobjective genetic algorithm is used to adjust the sextupole strengths in 12 families, giving two-sector translational symmetry.

Each longitudinal gradient dipole consists of five sections, where each section nominally has a constant dipole field. As discussed above, owing to the lack of transverse gradients, the sections are modeled as sector dipoles, with the edge focusing effects at the transitions between the sections properly described by the entrance and exit angles. Future work will improve on this simple model by incorporating the fact that these dipoles are in fact built from five straight segments, with both edge and body multipole content.

Magnets Q4, Q5, and Q8 provide negative bending, whereas M1, M2, M3, and M4 provide positive bending. The negative bending angle is $10.47 \%$ of the net bending angle. The natural emittance of the lattice is $41 \mathrm{pm}$ for the 

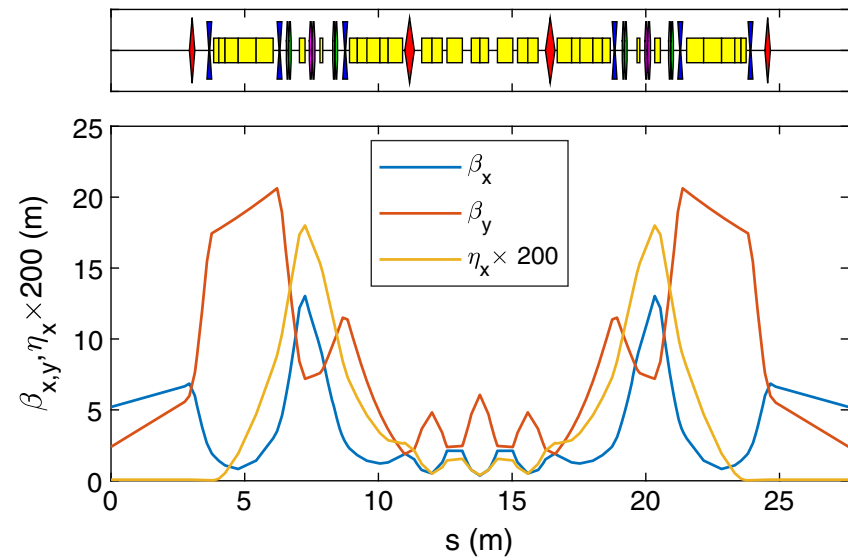

FIG. 4. Linear lattice functions for a sector of the APS-U lattice.

6-GeV ring. The beta and dispersion functions of one cell of the APS-U lattice is shown in Fig. 4.

In the following, the linear lattice parameters calculated with the two codes are compared first. This is followed by the tracking simulation of the dynamic aperture and the local momentum aperture. Straight geometry combinedfunction dipoles are modeled as described in Sec. II B. Quadrupole fringe fields with the calculated magnetic field profiles are included in the lattice model.

\section{A. Lattice parameters and linear optics}

Table III lists a few lattice parameters. The betatron tunes are different by only -0.0007 and 0.0007 for the horizontal and vertical planes, respectively, out of the total tunes of $\nu_{x}=95.1$ and $\nu_{y}=36.1$. This is an indication of the level of agreement in the linear optics modeling between

TABLE III. APS-U lattice parameters calculated with ELEGANT and AT.

\begin{tabular}{lrr}
\hline \hline Parameter & ELEGANT & \multicolumn{1}{c}{ AT } \\
\hline Horizontal tune, $\nu_{x}$ & 95.0999 & 95.0993 \\
Vertical tune, $\nu_{y}$ & 36.0999 & 36.1007 \\
Momentum compaction & 4.0406 & 4.0399 \\
$\quad$ MCF), $\times 10^{-5}$ & & \\
2nd-order MCF, $\times 10^{-4}$ & 1.2092 & 1.2091 \\
Chromaticity, $\xi_{x}$ & 8.1183 & 8.1704 \\
Chromaticity, $\xi_{y}$ & 4.7221 & 4.8739 \\
Natural chrom., $\xi_{x}$ & -133.6488 & -133.5874 \\
Natural chrom., $\xi_{y}$ nat & -111.6335 & -111.4689 \\
Emittance (pm) & 41.6612 & 41.6434 \\
Energy loss per turn $(\mathrm{MeV})$ & 2.8688 & 2.8700 \\
Momentum spread, $\sigma_{\delta}, \times 10^{-3}$ & 1.3499 & 1.3494 \\
Damping partition, $J_{x}$ & 2.2497 & 2.2495 \\
Damping time $\tau_{x}(\mathrm{~ms})$ & 6.8446 & 6.8424 \\
Horizontal tune, $\nu_{x}\left(\delta_{p}=0.04\right)$ & 95.4142 & 95.4410 \\
Vertical tune, $\nu_{y}\left(\delta_{p}=0.04\right)$ & 36.3556 & 36.3927 \\
Horizontal tune, $\nu_{x}(x=y=2 \mathrm{~mm})$ & 95.2313 & 95.2324 \\
Vertical tune, $\nu_{y}(x=y=2 \mathrm{~mm})$ & 36.1186 & 36.1189 \\
\hline \hline
\end{tabular}

the two codes. The momentum compaction factor and the beam parameters (e.g., emittance, momentum spread) are all given by the linear topics and they have similar agreement. Figure 5 shows the differences in a few linear optics functions between the two codes for one sector of the APS-U lattice. The fractional beta function difference, $\Delta \beta / \beta$, between the two codes is below $2 \times 10^{-4}$ and the difference in dispersion function is below $1.5 \mu \mathrm{m}$.

The calculation of chromaticity is an area where simulation codes tend to disagree [25]. In our study, the natural chromaticities and the corrected chromaticities for the horizontal and vertical planes agree very well between ELEGANT and AT. The natural chromaticities for the horizontal and vertical planes between AT and ELEGANT differ by 0.06 and 0.16 , out of -133 and -111 , respectively. The small relative differences indicate that the modeling of energy dependence in focusing elements is consistent in the two codes. The differences in the corrected chromaticities are 0.05 and 0.15 , respectively, which are nearly the same as the differences between the natural chromaticities. This is an indication that the energy dependence of particle motion in sextupole magnets is modeled consistently by the two codes, to a high degree of accuracy.
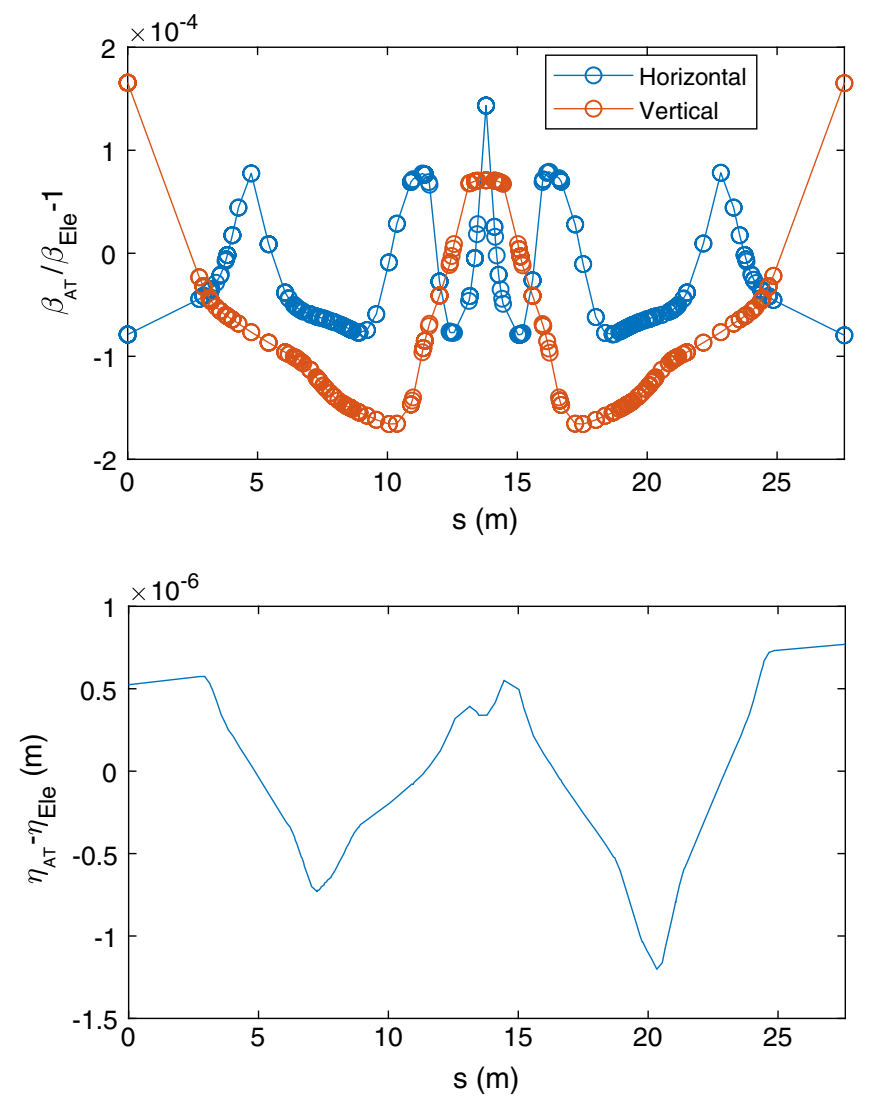

FIG. 5. Differences in linear optics functions between AT and ELEGANT for a sector of the APS-U lattice. Top: fractional beta function difference; bottom: dispersion function. 
TABLE IV. Changes of lattice parameters when the quadrupole fringe field is turned off in the APS-U lattce, calculated with ELEGANT and AT.

\begin{tabular}{lrr}
\hline \hline Parameter & ELEGANT & \multicolumn{1}{c}{ AT } \\
\hline Horizontal tune, $\Delta \nu_{x}$ & 0.0579 & 0.0582 \\
Vertical tune, $\Delta \nu_{y}$ & 0.1360 & 0.1374 \\
Chromaticity, $\Delta \xi_{x}$ & -0.0071 & -0.0688 \\
Chromaticity, $\Delta \xi_{y}$ & 0.6184 & 0.4942 \\
Horizontal tune, $\Delta \nu_{x}\left(\delta_{p}=0.04\right)$ & 0.1022 & 0.0690 \\
Vertical tune, $\Delta \nu_{y}\left(\delta_{p}=0.04\right)$ & 0.1792 & 0.1754 \\
Horizontal tune, $\Delta \nu_{x}(x=y=2 \mathrm{~mm})$ & 0.0738 & 0.0735 \\
Vertical tune, $\Delta \nu_{y}(x=y=2 \mathrm{~mm})$ & 0.1407 & 0.1415 \\
\hline \hline
\end{tabular}

Tables IV and V show, respectively the effects of the quadrupole fringe fields and the use of straight-pole dipoles instead of curved dipoles for Q4 and Q5. Of these, the quadrupole fringe fields have a more significant effect, particularly on the vertical tune and chromaticity. These values are not so large that they would prevent tuning of the ring if one did not know about them in advance. However, given the premium that will be placed on fast commissioning of the APS-U ring in order to return quickly to user operation, setting up the machine with knowledge of these effects is clearly beneficial. It is worth noting in this context that when these effects were added to the APS-U lattice, it provided necessary to reoptimize the nonlinear dynamics, since the linear optics cannot be exactly restored.

Initially, we found an apparently more significant effect for the straight-pole dipoles: the tunes changed in opposite directions by similar magnitudes, with $\Delta \nu_{x} \approx-0.3$ and $\Delta \nu_{y} \approx 0.2$. However, this was a result of tuning of the overall magnet strength to restore the trajectory, as described above. If we adjusted the dipole field only while holding the quadrupole component fixed, these differences drop to negligible values, as shown in the table.

\section{B. Nonlinear dynamics of the ideal lattice}

The ability to reliably predict the nonlinear beam dynamics performance by simulation codes is a key

TABLE V. Changes of lattice parameters when the APS-U straight dipoles are replaced by sector dipoles, calculated with ELEGANT and AT.

\begin{tabular}{lcc}
\hline \hline Parameter & ELEGANT & AT \\
\hline Horizontal tune, $\Delta \nu_{x}, \times 10^{-5}$ & -0.86 & -0.86 \\
Vertical tune, $\Delta \nu_{y}, \times 10^{-5}$ & 0.27 & 0.55 \\
Chromaticity, $\Delta \xi_{x}$ & -0.1187 & -0.0021 \\
Chromaticity, $\Delta \xi_{y}$ & 0.0791 & 0.0030 \\
$\Delta \nu_{x}\left(\delta_{p}=0.04\right), \times 10^{-3}$ & -6.46 & 0.68 \\
$\Delta \nu_{y}\left(\delta_{p}=0.04\right), \times 10^{-3}$ & 5.02 & 0.13 \\
$\Delta \nu_{x}(x=y=2 \mathrm{~mm}), \times 10^{-4}$ & 2.23 & -0.25 \\
$\Delta \nu_{y}(x=y=2 \mathrm{~mm}), \times 10^{-4}$ & -2.45 & -0.17 \\
\hline \hline
\end{tabular}

requirement in the lattice design of next generation synchrotron light sources. Accurate modeling of nonlinear beam dynamics is more challenging than modeling the linear optics because the nonlinear beam motion can be very sensitive to the initial conditions of the particles.

Because the betatron tunes differences between the two codes are very small, it is considered unnecessary to correct the betatron tunes in AT toward the ELEGANT values before making comparisons for nonlinear beam dynamics predictions. It was found that making tune corrections with the Q1 and Q2 quadrupole families causes larger beta function differences than the case without tune correction.

We first compared the nonlinear dynamics behaviors predicted by the two codes for the ideal lattice. For this comparison, physical apertures are removed in the ideal lattice in order to allow modeling the particle motion at large oscillation amplitudes. In Fig. 6, the on-energy horizontal and vertical phase space profiles are compared between the two codes. Particles with an initial horizontal or vertical position offset are launched and tracked for 1024 turns. All other initial coordinates are set to zero. The rf cavity is turned off in this simulation. The phase space profiles traced out by the two codes are very similar, despite the severe distortion introduced by nonlinearity at large offsets. The largest horizontal and vertical contours in Fig. 6 correspond to the extent of the stable phase space area in the two transverse planes (while the action in the other plane is zero), respectively. The beta functions are $\beta_{x}=5.20 \mathrm{~m}$ and $\beta_{y}=$ $2.39 \mathrm{~m}$ at the launching point. The islandlike structures in
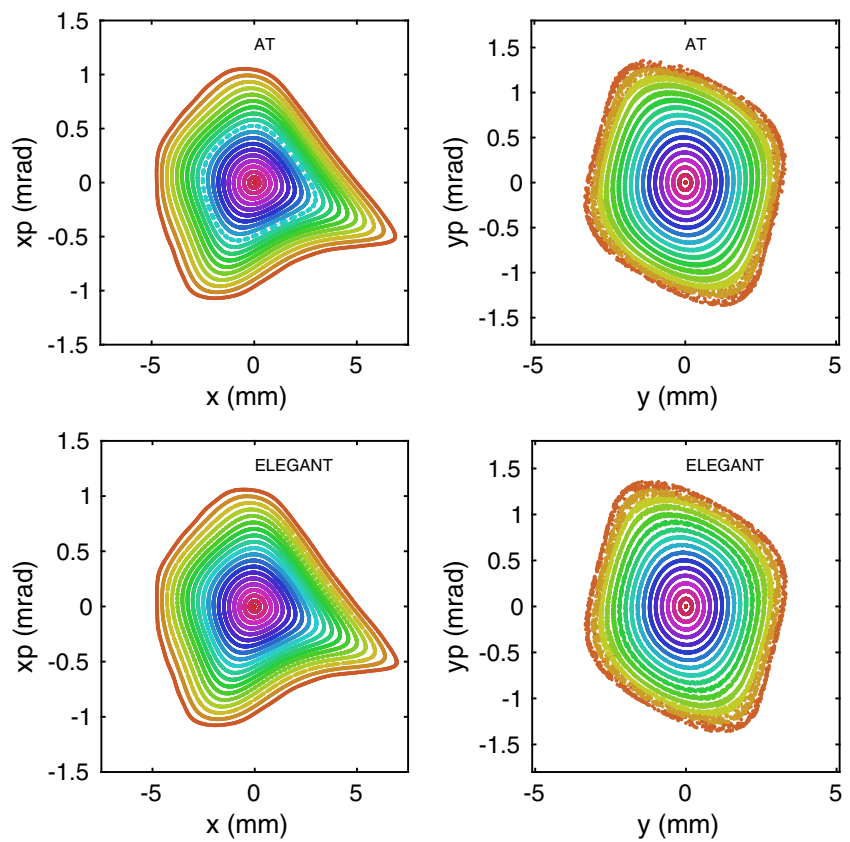

FIG. 6. Comparison of Hamiltonian contours in the horizontal phase space by tracking simulation for ELEGANT and AT. $\beta_{x}=$ $5.20 \mathrm{~m}$ and $\beta_{y}=2.39 \mathrm{~m}$ at the launching point. $\mathrm{Rf}$ cavity is turned off in the model. Top: AT; bottom: ELEGANT. 


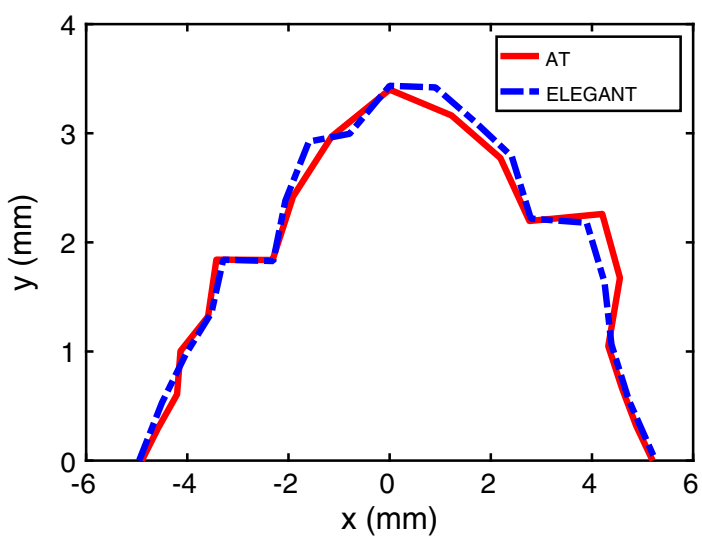

FIG. 7. Comparison of DA found by the two codes (ELEGANT and AT) for the ideal APS-U lattice, without physical apertures, radiation, or rf cavity.

the horizontal phase space plot by ELEGANT indicate horizontal tune values that are very close to the low-order fraction numbers. The lack of such structures in the AT plot is due to the small tune difference of -0.0007 .

Figure 7 compares the dynamic aperture (DA) of the ideal lattice determined by the two codes. Physical apertures, $\mathrm{rf}$ cavities, and radiation effects are all absent in the lattice model for this comparison. DA is determined by
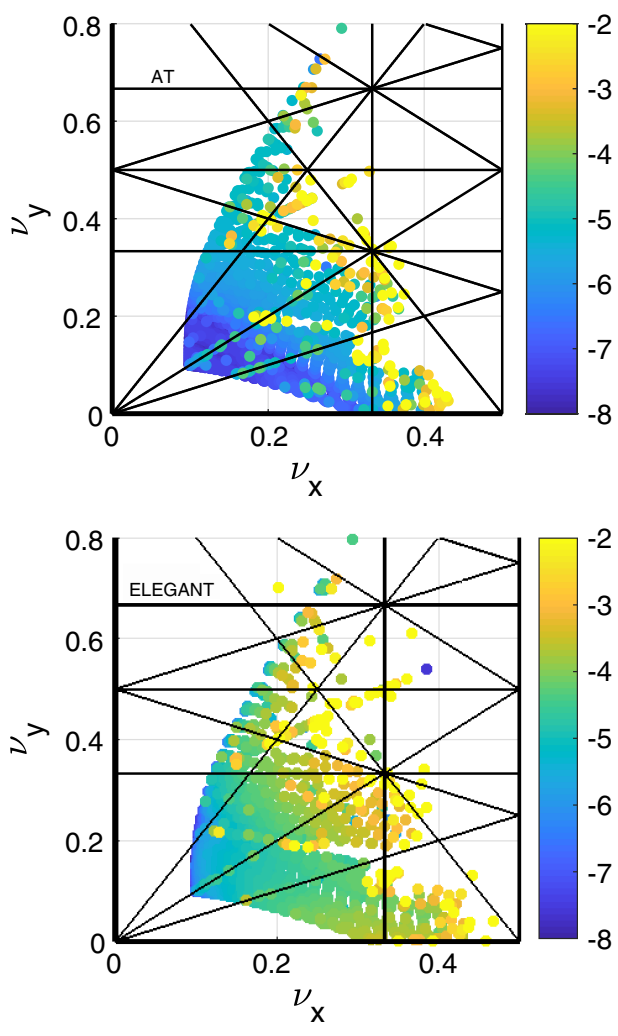

FIG. 8. Tune diagram for frequency map analysis in the $x-y$ plane. The color code represents the detuning over 1024 turns, $\log _{10}\left(\sqrt{\Delta \nu_{x}^{2}+\Delta \nu_{y}^{2}}\right)$, where $\Delta \nu_{x}$ and $\Delta \nu_{y}$ are tune changes from the first 512 turns to the second 512 turns. launching particles with initial position offset on the $x-y$ plane and track for 1024 turns. The initial positions of the particles are on 19 rays extending from the origin. The DA boundary on each ray is given by the last surviving particle from the origin outward. The DA found by the two codes is nearly the same, except for small deviations in the upper right corner.

Frequency map analysis (FMA) [37] was also conducted for the ideal lattice. Particles on a dense grid in the $x-y$ plane were tracked for 1024 turns. The tune diffusion, defined as the combined betatron tune shifts with time,

$$
\text { diffusion } \equiv \log _{10} \sqrt{\Delta \nu_{x}^{2}+\Delta \nu_{y}^{2}}
$$

was evaluated by computing the tune changes, $\Delta \nu_{x}$ and $\Delta \nu_{y}$, between the first and second 512 turns. Figure 8 shows the comparison of tune diffusion rate in the tune diagram between AT and ELEGANT. The tune footprint is nearly identical between the two codes. The differences in the diffusion seem to represent real differences between the two codes.

We also performed FMA in the $x-\frac{\Delta p}{p}$ plane. The momentum deviation coordinate was varied from $-4.5 \%$ to $4.5 \%$ with the step size of $0.25 \%$. Tune diffusion over the $x-\frac{\Delta p}{p}$ plane is plotted in Fig. 9. While again there is some difference in the evaluation of the tune diffusion,
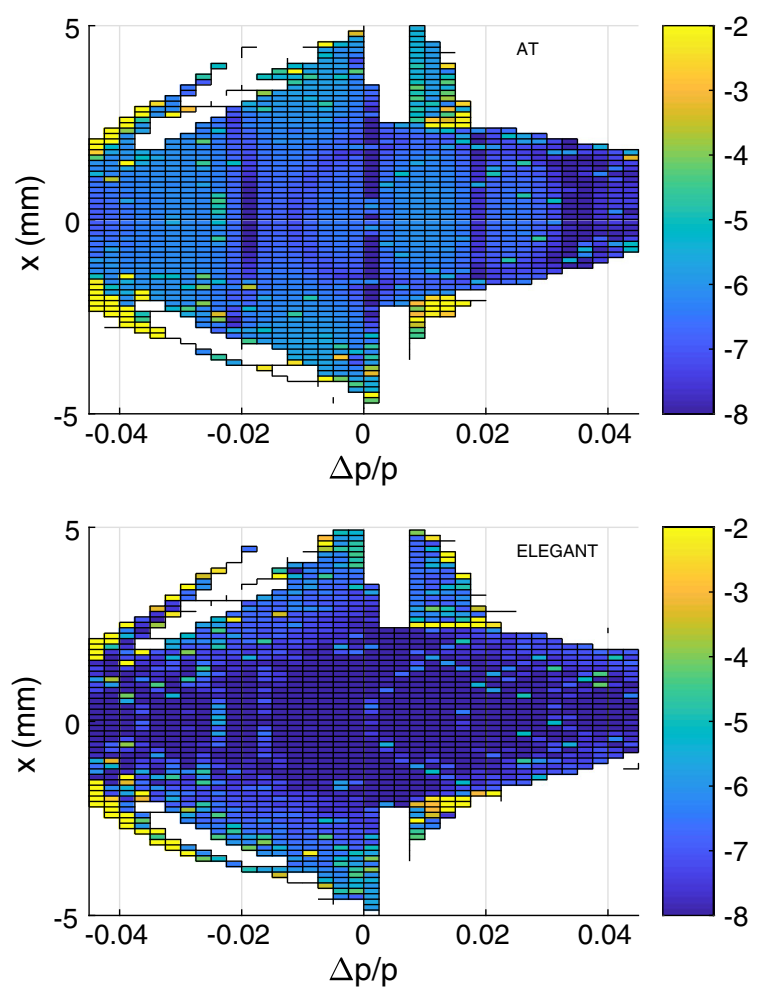

FIG. 9. Comparison of stability region in the $x$ - $\frac{\Delta p}{p}$ plane as calculated by AT (top) or ELEGANT (bottom). Color code represents tune diffusion as defined in Eq. (13). 


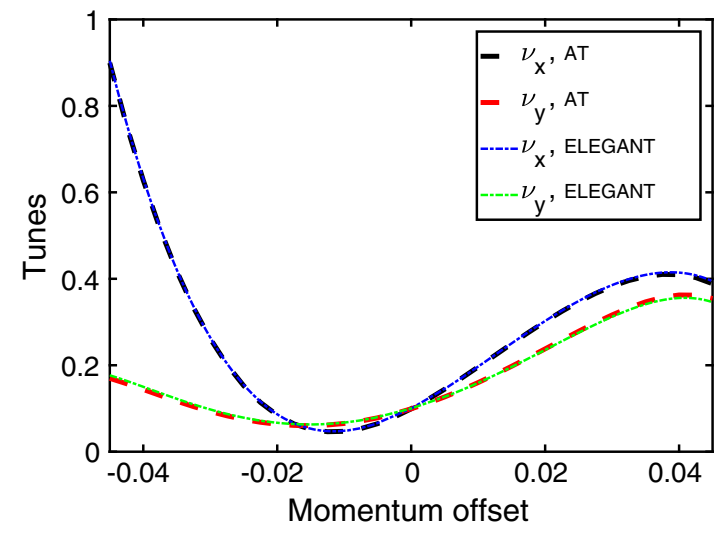

FIG. 10. Comparison of betatron tunes as a function of momentum deviation calculated by AT and ELEGANT.

the stability region in the $x-\frac{\Delta p}{p}$ plane calculated by the two codes is very similar.

The dependence of betatron tunes over the momentum deviation is often used to characterize the off-energy dynamics performance of a lattice. Betatron tunes vs. momentum deviation for the APS-U lattice calculated by both codes are compared in Fig. 10. Within a large range of momentum errors, the tunes agree between the two codes to high accuracy. This indicates that the codes not only model the linear chromaticities in a consistent manner, but also the high order chromaticities.

In summary, comparison of basic nonlinear beam dynamics behavior for the ideal APS-U upgrade lattice calculated by AT and ELEGANT shows that the two codes are in very good agreement in the prediction of both geometric and chromatic behaviors within the full stability region of the lattice.

\section{DA and LMA for lattices with linear errors}

A workable lattice has to be able to deliver the required dynamic aperture and momentum aperture when a certain level of lattice errors is present because a realistic machine always has errors. The robustness of a lattice is typically checked by generating an ensemble of perturbed lattices, each with a different set of random errors, and evaluating the variation of DA and LMA among the ensembles. As a part of the code validation study, we performed this process for the APS-U lattice with both AT and ELEGANT.

We generated 25 perturbed lattices by introducing small random quadrupole and skew quadrupole errors to the sextupole magnets in the lattice. The level of quadrupole errors was chosen such that the horizontal and vertical beta beating (rms) were both on the order of $1 \%$. The level of skew quadrupole errors gives an emittance ratio of $\sim 10 \%$ when the horizontal and vertical tunes are shifted apart by 0.1 (with fractional part of $\nu_{x}$ and $\nu_{y}$ at 0.05 and 0.15 , respectively). When the betatron tunes are restored, the ratio is $\sim 100 \%$.

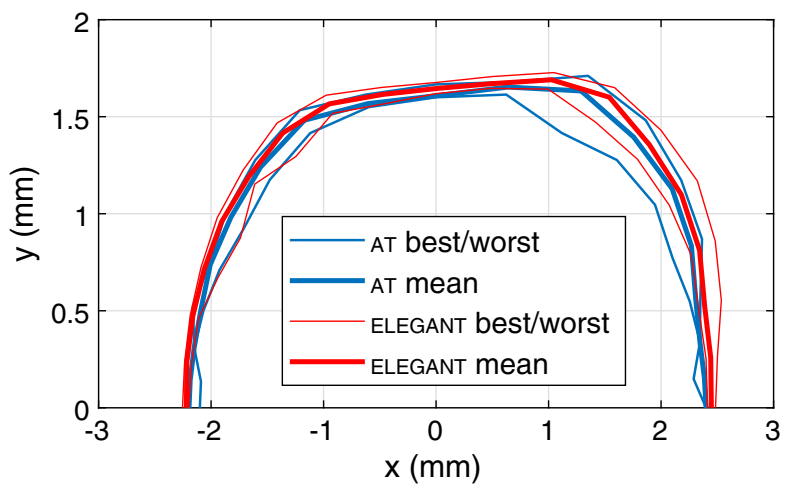

FIG. 11. Dynamic apertures with the same 25 error seeds evaluated by AT and ELEGANT are compared. The thin curves show the average DA of the 25 seeds, while the thin curves show the best and worst of all seeds. Tracking is done with physical apertures and radiation effects by bends. Rf voltage is at $4.8 \mathrm{MV}$.

The DA and LMA are evaluated by particle tracking simulation in a realistic manner. The $352-\mathrm{MHz}$ main $\mathrm{rf}$ cavities are turned on, with the total $\mathrm{rf}$ voltage set to 4.8 MV. Radiation damping is simulated by losing a proper amount of energy at each integration step in the dipole magnets for the particles. All the physical apertures are in the lattice model. The elliptic aperture passmethod is
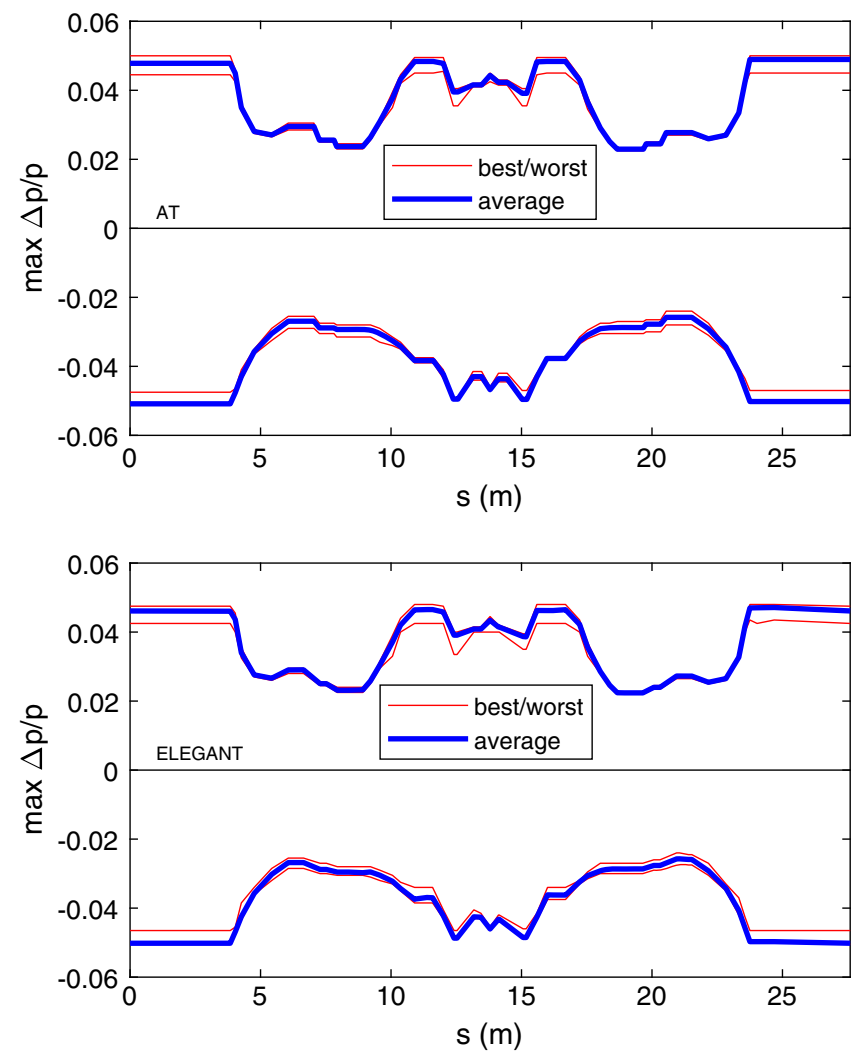

FIG. 12. Local momentum aperture for the first sector of the APS-U lattice with 25 random error seeds obtained with particle tracking by AT (top) or ELEGANT (bottom). 
implemented in AT. A special aperture type, the "speed bump" aperture, is also implemented in AT using the same physics model as in ELEGANT [38]; compared to a simple flat aperture, this method more accurately simulates the planned collimators in the high-dispersion region that are used to intercept Touschek-scattered particles.

The DA is determined by launching particles on 19 rays and tracking for 1024 turns, as described earlier for the ideal lattice. The average DAs of the 25 lattices found by the two codes are shown in Fig. 11, along with the best and worst cases. There is good agreement except for a small difference at the upper right corner, which corresponds to the unstable region around $\nu_{x}=0.2$ and $\nu_{y}=0.2$ in Fig. 8 .

The LMA is determined by launching particles with initial energy errors, ranging from $\delta=-0.06$ to 0.06 , with step size of 0.0005 and tracking for 2048 turns from each location of interest. The locations of interest include the entrance and exit faces of all dipole magnets and the entrance points of all magnets in the first sector. Figure 12 shows the distribution of LMA on the positive and negative sides over the length of the first sector for AT (top) and ELEGANT (bottom). The results are remarkably close.

\section{CONCLUSION}

In order to accurately model the nonlinear beam dynamics performance of next-generation storage ring light sources, new features were introduced into the lattice modeling codes ELEGANT and AT in two separate, independent efforts. A new development in the codes is the proper modeling of combined-function quadrupole-bend magnets on a straight geometry. The linear and nonlinear effects of quadrupole fringe fields are also included in the lattice model. Quadrupole fringe field effects were previously modeled in ELEGANT and AT; but typically they are not included in initial lattice designs.

We used the APS-U lattice to benchmark the two codes and found excellent agreement in both linear optics calculations and the prediction of nonlinear beam dynamics behaviors. The results we present here boost our confidence in making accurate predictions of lattice performance with precise modeling codes and encourage further refinement.

\section{ACKNOWLEDGMENTS}

This work was supported by the U.S. Department of Energy, Office of Science, Office of Basic Energy Sciences, under Contract No. DE-AC02-76SF00515 (SLAC) and under Contract No. DE-AC02-06CH11357 (ANL).

[1] M. Borland, V. Sajaev, L. Emery, and A. Xiao, in Proceedings of the 23rd Particle Accelerator Conference, Vancouver, Canada, 2009 (IEEE, Piscataway, NJ, 2009), pp. 3850-3852.
[2] L. Yang, Y. Li, W. Guo, and S. Krinsky, Multiobjective optimization of dynamic aperture, Phys. Rev. Accel. Beams 14, 054001 (2011).

[3] X. Huang and J. Safranek, Nonlinear dynamics optimization with particle swarm and genetic algorithms for SPEAR3 emittance upgrade, Nucl. Instrum. Methods Phys. Res., Sect. A 757, 48 (2014).

[4] X. Huang and J. Safranek, Online optimization of storage ring nonlinear beam dynamics, Phys. Rev. Accel. Beams 18, 084001 (2015).

[5] S. Liuzzo, N. Carmignani, L. Farvacque, B. Nash, T. Perron, P. Raimondi, R. Versteegen, and S. M. White, in Proceedings of IPAC2016 (IEEE, Busan, Korea, 2016), pp. 3420-3422.

[6] D. K. Olsson, in Proceedings of IPAC2018 (Vancouver, BC, Canada, 2018) pp. 2281-2283.

[7] X. Yang, G. Ganetis, Y. Hidaka, T. Shaftan, V. Smaluk, G. Wang, L.-H. Yu, and P. Zuhoski, in Proceedings of IPAC2019 (Melbourne, Australia, 2019).

[8] Advanced Photon Source Upgrade Project Preliminary Design Report, Argonne National Laboratory, Report No. aPSU-2.01-RPT-002, 2017.

[9] L. Farvacque, N. Carmignani, J. Chavanne, A. Franchi, G. L. Bec, S. Liuzzo, B. Nash, and T. P. P. Raimondi, in Proceedings of the 4th International Particle Accelerator Conference, IPAC-2013, Shanghai, China, 2013 (JACoW, Shanghai, China, 2013), pp. 79-81.

[10] C. Steier et al., in Proceedings of IPAC 2015 (IEEE, Richmond, VA, USA, 2015), pp. 1840-1842.

[11] J. Guo and T. Raubenheimer, in Proceedings of the 8th European Particle Accelerator Conference, Paris, 2002 (EPS-IGA and CERN, Geneva, 2002), p. 1136.

[12] Y. Papaphilippou and P. Elleaume, in Proceedings of the 21st Particle Accelerator Conference, Knoxville, TN, 2005 (IEEE, Piscataway, NJ, 2005), p. 2086.

[13] R. Nagaoka and A.F. Wrulich, Emittance minimisation with longitudinal dipole field variation, Nucl. Instrum. Methods Phys. Res., Sect. A 575, 292 (2007).

[14] C.-x. Wang, Minimum emittance in storage rings with uniform or nonuniform dipoles, Phys. Rev. Accel. Beams 12, 061001 (2009).

[15] A. Streun and A. Wrulich, Compact low emittance light sources based on longitudinal gradient bending magnets, Nucl. Instrum. Methods Phys. Res., Sect. A 770, 98 (2015).

[16] J. Delahaye and J. Potier, in Proceedings of the 1989 Particle Accelerator Conference, Chicago, IL (IEEE, New York, 1989), p. 1611.

[17] A. Streun, The anti-bend cell for ultralow emittance storage ring lattices, Nucl. Instrum. Methods Phys. Res., Sect. A 737, 148 (2014).

[18] M. Yoon, J. Corbett, M. Cornacchia, J. Tanabe, and A. Terebilo, Analysis of a storage ring combinedfunction magnet: trajectory calculation and alignment procedure, Nucl. Instrum. Methods Phys. Res., Sect. A 523, 9 (2004).

[19] X. Huang, J. Safranek, and D. Dell' Orco, in Proceedings of the International Particle Accelerator Conference, Kyoto, Japan (ICR, Kyoto, 2010), p. 4626. 
[20] Y. Li and X. Huang, A practical approach to extract symplectic transfer maps numerically for arbitrary magnetic elements (2015), https://arxiv.org/pdf/1511.00710.pdf.

[21] G. E. Lee-Whiting, End effects in first-order theory of quadrupole lenses, Nucl. Instrum. Methods 76, 305 (1969).

[22] J. Irwin and C. x Wang, in Proceedings of the Particle Accelerator Conference, Dallas, TX, 1995 (IEEE, New York, 1995), pp. 2376-2378.

[23] D. Zhou, J. Tang, Y. Chen, and N. Wang, in Proceedings of the International Particle Accelerator Conference, Kyoto, Japan (ICR, Kyoto, 2010), pp. 4500-4502.

[24] E. Forest and J. Milutinovic, Leading order hard edge fringe fields effects exact in $(1+\delta)$ and consistent with Maxwell's equations for rectilinear magnets, Nucl. Instrum. Methods Phys. Res., Sect. A 269, 474 (1988).

[25] D. Einfeld, Summary of code comparison, https://www .diamond.ac.uk/Home/Events/2009/NBD_workshop.html (2009), nonlinear Beam Dynamics Workshop.

[26] M. Borland, elegant: A flexible sdds-compliant code for accelerator simulation, Advanced Photon Source Report No. LS-287, September 2000.

[27] A. Terebilo, in Proceedings of the 19th Particle Accelerator Conference, Chicago, IL, 2001 (IEEE, Piscataway, NJ, 2001), pp. 3203-3205.

[28] L. C. Teng, Expanded Form of Magnetic Field with Median Plane, Technical Report No. LCT-28, ANL, 1962.
[29] K. L. Brown, A first- and second-order matrix theory for the design of beam transport systems and charged particle spectrometers, SLAC Technical Report No. 75, 1982.

[30] E. Forest, Beam Dynamics: A New Attitude and Framework, The Physics and Technology of Particle and Photon Beams Vol. 8 (Hardwood Academic/CRC Press, Amsterdam, The Netherlands, 1998).

[31] M. Borland, Symplectic integration in elegant, Argonne National Laboratory, Tech. Rep. No. ANL/APS/LS-356, 2019.

[32] K. Hwang and S. Y. Lee, Dipole fringe field thin map for compact synchrotrons, Phys. Rev. Accel. Beams 18, 122401 (2015).

[33] M. Venturini and A. J. Dragt, Accurate computation of transfer maps from magnetic field data, Nucl. Instrum. Methods Phys. Res., Sect. A 427, 387 (1999).

[34] G. E. Lee-Whiting, Third-order aberrations of a magnetic quadrupole lens, Nucl. Instrum. Methods 83, 232 (1970).

[35] C.X. Wang (private communication).

[36] M. Borland, A High-Brightness Thermionic Microwave Electron Gun, Technical Report No. 402, SLAC, 1991.

[37] J. Laskar, Frequency analysis for multi-dimensional systems. Global dynamics and diffusion, Physica D (Amsterdam) 67, 257 (1993).

[38] Elegant online manual, https://ops.aps.anl.gov/manuals/ elegant_latest/elegant.html. 